\title{
A balanced whole arm reciprocal translocation resulting in three different adverse pregnancy outcomes
}

\author{
P J Cooper, C Towe, J A Crolla
}

\begin{abstract}
A family with a whole arm translocation $t(13 ; 18)(13 p 18 p ; 13 q 18 q)$ was ascertained through a stillbirth with clinical features suggestive of trisomy 18 . Two subsequent pregnancies both spontaneously aborted; one was found to have a $69, \mathrm{XXX}, \mathrm{t}(13 ; 18) \mathrm{mat}$ chromosome constitution while the other had a $45, X Y,-13,-18,+\operatorname{der}(18) t(13 ; 18)$ mat chromosome constitution. (f Med Genet 1993;30:417-18)
\end{abstract}

\section{Case reports}

The proband was a 34 week male stillbirth with intrauterine growth retardation, unilateral left cleft lip, total cleft palate, rocker bottom feet, and a large patent ductus and foramen ovale. Unfortunately the tissue from the stillbirth failed to grow. Examination of the parents' blood lymphocytes showed the mother (figure, II.2) to be the carrier of a balanced reciprocal whole arm translocation between chromosomes 13 and $18,46, \mathrm{XX}$, $t(13 ; 18)(13 p 18 p ; 13 q 18 q)$, while the father (II-1) had an apparently normal male karyotype. The chromosomes of the mother's parents were normal. As the clinical features of the stillbirth were reminiscent of those of trisomy 18 it was presumed that adjacent 2 segregation in the mother (see also Discussion) gave rise to a $46, X Y,-13,+\operatorname{der}(18) t(13 q 18 q)$ karyotype in the stillbirth resulting in trisomy for the long arm of chromosome 18 .

A second pregnancy (III.2) spontaneously

Wessex Regional Genetics Laboratory, Salisbury District Hospital, Odstock, Salisbury SP2 8BJ UK.

P J Cooper

C Towe

$\mathrm{J}$ A Crolla

Correspondence to Dr Crolla.

Received 7 April 1992. Revised version accepted 15 October 1992.
II

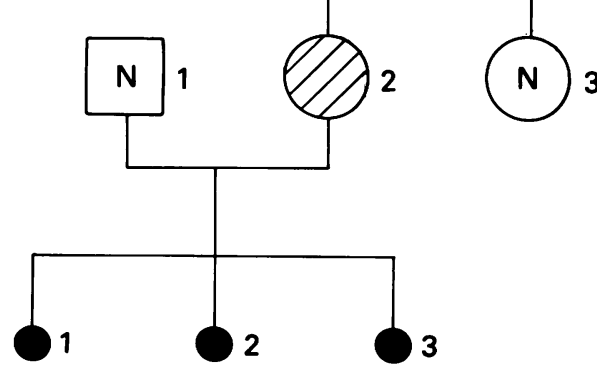

Pedigree. $I I \cdot 2=46, X X, t(13 ; 18)(q 11 ; p 11)$ de novo. $I I I \cdot 1=46, X Y,-13,+\operatorname{der}(18) t(13 ; 18)(q 11 ; p 11)$ mat (assumed). III.2 $=69, X X X, t(13 ; 18)(q 11 ; p 11)$ mat . $I I I \cdot 3=45, X Y,-13,-18,+\operatorname{der}(18) t(13 ; 18)$ (q11;p11) mat. $N=$ Chromosomally normal.

aborted in the first trimester. A small intact amniotic sac containing a non-molar, $3 \mathrm{~mm}$, resorbing, disorganised fetus was referred to the laboratory. Karyotyping showed triploidy with the balanced translocation also present, $69, \mathrm{XXX}, \mathrm{t}(13 ; 18)$. In situ hybridisation using a chromosome 18 centromere specific probe (D18Z1) showed the centromere of chromosome 18 to be present on the larger, but not the smaller, of the translocation products. Thus the translocation can be more accurately described as $t(13 ; 18)(q 11 ; p 11)$. Studies of the chromosome heteromorphisms of the fetus and both parents using both $\mathrm{C}$ and $\mathrm{Q}$ banding showed six chromosomes to be informative with respect to the origin of the triploidy. They showed that triploidy could only have arisen as a result of an error of the first maternal meiotic division.

The third pregnancy (III-3), also a first trimester spontaneous abortion, produced a small ruptured empty amniotic sac. Chromosome analysis showed an unbalanced translocation arising as a result of 3:1 segregation, giving a karyotype of $45, \mathrm{XY},-13,-18$, $+\operatorname{der}(18) t(13 ; 18)$.

\section{Discussion}

It is well established that if the quadrivalent in the gametocyte at meiosis I is substantially asymmetrical with one of the chromosomes being small, as in this case, 3:1 disjunction is the most likely form of malsegregation. ${ }^{1}$ Indeed, the third pregnancy (III-3) was found to be a result of 3:1 disjunction.

If, however, the malformed fetus observed in the first pregnancy (III-1) was the result of an adjacent 2 segregation this is also not totally unexpected. A predisposition to adjacent 2 segregation is associated with translocations in which the sum of the size of the translocated segments is greater than the sum of the size of the centric segments. ${ }^{1}$ Obviously in this case the combination of $13 q$ and $18 p$ is greater than

$13 \mathrm{p}$ and 18q. However, Jalbert et al ${ }^{1}$ also commented on the 'almost mutual exclusiveness' of the three modes of malsegregation. Of 151 reciprocal translocations involved in their study only five displayed two types of segregation, all of these sharing adjacent 1 and $3: 1$ types. If the stillbirth (III-1) was indeed a result of adjacent 2 segregation then this case would appear to be very unusual. However, other possibilities exist; 3:1 segregation in this translocation could produce interchange trisomy for chromosome 13 or 18 . The proband's clinical features, although suggestive of tri- 
somy 18 , do not rule out the possibility of trisomy 13 and therefore both interchange trisomies are feasible.

Trisomies and partial monosomies of chromosomes 13 and 18 often survive to term and it is therefore not unexpected to find different aneuploid segregants of a translocation involving these chromosomes surviving long enough to produce a clinically recognised pregnancy.

We know of only three previous reports of triploidy in a balanced translocation carrier all of which were shown to have arisen most probably as a result of errors at the first meiotic division (two paternal and one maternal in origin). ${ }^{23}$ Thus it seems likely that all four triploid fetuses carrying a balanced translocation have resulted from an error of the first meiotic division, and this suggests that the presence of a translocation may predispose to a failure of the first meiotic division of the gamete.

While the increased risk of pregnancy loss in translocation carriers is well established and assumed to be the result of chromosomally unbalanced gametes resulting in a non-viable pregnancy, there are remarkably few reported cases where the fetal chromosomes have actually been examined. Our report suggests that translocations could be responsible for pregnancy losses not only as the result of aneuploid segregants but also by secondly causing other types of chromosome abnormality.

We thank P A Jacobs, A Cockwell, E Smith, and all the staff at the Wessex Regional Genetics Laboratory for their assistance, patience, and support.

1 Jalbert P, Sele B, Jalbert H. Reciprocal translocations: a way to predict the mode of imbalanced segregation by pachy tene-diagram drawing. A study of 151 human translocations. Hum Genet 1980;55:209-22.

2 Jacobs PA. The origin of chromosome abnormalities in man In: Chromosomes today. Vol 7. 7th International Chromosome Conference, Oxford. London: George Allen \& Unwin, 1981:271-80.

3 Rochon L, Vekemans MJJ. Triploidy arising from a first meiotic non-disjunction in a mother carrying a reciprocal translocation. F Med Genet 1990;27:724-6. 